\title{
Planeación de la capacidad hospitalaria: un enfoque desde el flujo de pacientes con Dinámica de Sistemas
}

\section{Capacity planning in hospitals: an approach from patients flow with System Dynamics}

\author{
DOI: http://doi.org/10.17981/ingecuc.16.1.2020.16
}

Artículo de Investigación Científica. Fecha de Recepción: 30/09/2019. Fecha de Aceptación: 03/02/2020

\author{
Edgar Leonardo Duarte Forero (1) \\ Universidad Libre. Bogotá, D.C. (Colombia) \\ edgarl.duartef@unilibre.edu.co \\ Manuel Ángel Camacho Oliveros (it) \\ Universidad Libre. Bogotá, D.C. (Colombia) \\ manuel.camachoo@unilibre.edu.co
}

Para citar este artículo:

E. Duarte Forero, M. Camacho Oliveros, "Planeación de la capacidad hospitalaria: un enfoque desde el flujo de pacientes con Dinámica de Sistemas”, INGE CUC, vol. 16, no. 1, pp. 217-233, 2020. DOI: http://doi.org/10.17981/ingecuc.16.1.2020.16

\begin{abstract}
Resumen
Introducción- La adecuada administración de los recursos disponibles es un factor que incide significativamente en la calidad de la atención en hospitales. Las nuevas tecnologías en salud, el envejecimiento de la población, y la escasa disponibilidad presupuestal conllevan a mejorar la administración de la capacidad hospitalaria para lograr mejores desempeños globales.

Objetivo- Construir herramientas que permitan estudiar los factores que inciden en los indicadores de eficiencia hospitalaria global (giro-cama, porcentaje ocupacional y estancia promedio) desde la perspectiva del flujo de pacientes.

Metodología- Se desarrolló un estudio utilizando Dinámica de sistemas en un hospital público de alta complejidad desde la perspectiva del flujo de pacientes, haciendo énfasis en la parametrización de condicionantes de capacidad y en la medición del impacto en indicadores de eficiencia global. La exploración de estrategias se desarrolló con análisis de varianzas y análisis de componentes principales.

Resultados- El porcentaje ocupacional reflejó mayor sensibilidad al uso de políticas sobre uso de camas y reducción de tiempos de espera. El giro cama fue el indicador con mayor velocidad de respuesta a los cambios implementados. El aumento de las horas-médico disponibles en urgencias no presentó impactos significativos en el flujo de pacientes ni en las medidas de desempeño global.

Conclusiones- La simulación continua y análisis de componentes principales permitió obtener herramientas para la planeación de capacidad en el mediano y largo plazo. En lugar de invertir en el aumento de capacidad instalada, la gerencia debe priorizar la reducción de desperdicios a través de metodologías como Lean healthcare.

Palabras clave- Dinámica de sistemas; flujo de pacientes; logística hospitalaria; giro cama; porcentaje ocupacional; hospital; análisis de componentes principales
\end{abstract}

\begin{abstract}
Introduction- An adequate Resource management strategy influences significantly on Quality of service in hospitals. New technologies in healthcare, population ageing and lack of founding drive to continuous improvements in hospitals' capacity management in order to achieve a better performance of itself.

Objective- To develop tools that may allow a deeper understanding of those drivers that influence global hospital efficiency (bed turnover, bed occupancy, length of stay) from the point of view of patients flow.

Methodology - A research based on Systems Dynamics was developed at a high complexity public hospital, emphasizing in the perspective of patients flow, the definition of parameters that influence capacity and the measurement of the impact of improvement strategies in global efficiency indicators. The study of strategies was based on Analysis of Variance and Principal component analysis (PCA).

Results- Bed occupancy showed higher sensibility than other Key Performance Indicators (KPI) after the implementation of policies regarding the use of beds and the reduction of waiting times. Bed turnover was the KPI with the fastest reaction after new policies implementation. Improvement in medical staff capacity at Emergency Rooms did not display significative impacts in patients flow nor global hospital efficiency.

Conclusions- Continuous simulation and PCA worked together to brought functional tools for capacity planning in middle and long terms. Investments should be dedicated to reduction of wasting through methodologies such as Lean Healthcare, rather than increasing installed capacity.

Keywords- System dynamics; Patient Flow, Healthcare Logistics; Bed turnover; Bed occupancy; Hospital; Principal component analysis
\end{abstract}




\section{INTRODUCCIÓN}

La gestión de la capacidad en el uso de recursos físicos es un factor de gran complejidad para la administración de instituciones hospitalarias. Por una parte, mientras la gerencia aspira a lograr altos niveles de utilización de su capacidad, la variabilidad en la demanda y la incertidumbre en los tiempos de atención puede hacer que estos recursos no estén disponibles cuando se requieren o que se subutilicen [1]. Las principales causas de esta disonancia residen en una baja capacidad instalada en los recursos, una inadecuada programación en su uso, o demoras en los flujos de información [2, p. 183].

El estudio de la gestión de la capacidad en instituciones hospitalarias constituye además un reto en tanto que se trata de la prestación de un servicio y no de un bien material [3]. Los servicios puros son intangibles, con alta carga de trabajo, heterogéneos, no pueden ser almacenados o transportados y su consumo ocurre simultáneamente con su producción [4]. Adicionalmente, son altamente influenciados por el consumidor y su calidad es muy difícil de juzgar [5, p. 2].

La gestión de la prestación de servicios de salud requiere de adaptaciones específicas de las herramientas que tradicionalmente se han aplicado en ambientes de manufactura. La propuesta de Niranjan y Weaver [6, p. 2407] presenta la comparación de los conceptos de inventario, capacidad, tiempos de ciclo, amplificación de la variabilidad, desperdicios y materias primas entre cadenas de suministro de bienes y de servicio. En su trabajo, los autores destacan que el concepto de inventario de bienes se debe asumir en las cadenas de servicio como la capacidad disponible (mano de obra, equipos, instalaciones) para satisfacer los requerimientos del cliente. En el plano de los servicios de salud, Dobrzykowski et al. [7] presentan un análisis sistemático sobre las contribuciones desde la investigación en manufactura y cadenas de suministros hacia la gestión hospitalaria y de salud.

El enfoque de las camas hospitalarias es uno de los más utilizados para el análisis de la gestión de capacidad en hospitales [8]. Esto se fundamenta en que alrededor de la cama hospitalaria se concentran servicios de cuidado de enfermería, atención médica, hotelería y traslado de pacientes, entre otros. No obstante, esta perspectiva basada en la gestión de las camas no ofrece una visión completa de los servicios que se ofertan al interior del hospital, dado que la atención es concentrada en un solo tipo de activos, dejando de lado el uso de otros recursos como el humano, tecnológico y de infraestructura [9]. Adicionalmente, la conjugación de servicios ofertados a quien ocupa la cama genera una mezcla de costos muy difícil de identificar, así como dificultades en la predicción de la demanda [10].

En la práctica, la gestión de capacidad hospitalaria puede ser medida desde múltiples puntos de vista: calidad, seguridad, eficiencia o eficacia [11]. El presente trabajo se enfoca en el análisis de los tres indicadores básicos de eficiencia hospitalaria global: giro cama, porcentaje ocupacional y tiempo de estancia [12, p. 208]. El giro cama hace énfasis en la eficiencia del uso del recurso cama: representa el número de pacientes que utilizan una misma cama en un periodo de tiempo. El porcentaje ocupacional examina la cantidad de pacientes alojados durante un periodo con respecto al número de camas disponibles en ese mismo periodo. Finalmente, el tiempo de estancia se orienta hacia la eficiencia del uso de todos los recursos disponibles, en tanto que no se restringe específicamente a las camas sino al resultado total de la gestión de recursos: el número de días en que un paciente estuvo interno en el hospital [13]. A continuación, se presentan los mecanismos de cálculo respectivos. Una ampliación de la relación entre estos tres indicadores puede ser encontrada en el artículo seminal de Pabón Lasso [14].

$$
\begin{aligned}
& \text { Porcentaje ocupacional }=\frac{\text { Días cama ocupados }}{\text { Días cama instaladas }} * 100 \\
& \text { Estancia Promedio }=\frac{\text { Días cama pacientes egresados }}{\text { Total egresos }} * 100 \\
& \text { Giro cama }=\frac{\text { Total egresos }}{\text { Días cama instaladas }}
\end{aligned}
$$


Partiendo de las necesidades de gestionar la capacidad hospitalaria como un servicio, y de evaluar su desempeño a través de los tres indicadores básicos (giro cama, porcentaje ocupacional y tiempo de estancia), surge la cuestión acerca de cómo hacerlo. Una perspectiva utilizada para ello está sustentada por Rechel [9, p. 634] cuyo trabajo propone examinar el flujo de pacientes como una estrategia para obtener un mejor uso de los recursos hospitalarios. Más allá de estudiar la ocupación de las camas instaladas, el autor propone el análisis de las rutas que experimentan los pacientes en ambientes intrahospitalarios de manera agregada. El análisis de estas rutas a través del estudio de su flujo, proviene de la adaptación de estrategias de manufactura esbelta (Lean Manufacturing) en ambientes hospitalarios, cuya perspectiva supone que los hospitales no deben ser vistos como "almacenes" sino como "plantas de producción de servicios" con procesos inmensamente complejos en donde la identificación de los cuellos de botella ayuda a brindar mejoras al throughput total de la organización [9].

Las herramientas Lean tienen origen en la mejora continua de procesos de fabricación y buscan continuamente eliminar los desperdicios en el uso de la capacidad [15]. En la medida en que se aumente el flujo medido a través del indicador throughput (número de unidades producidas por unidad de tiempo), se estará alcanzando en mayor medida la expectativa del cliente. Con el uso de herramientas Lean, las instituciones hospitalarias pueden lograr mayores eficiencias en el uso de sus recursos, no solamente en áreas como urgencias, sino también en hospitalización, imágenes diagnósticas, almacenes y recursos humanos [16], [17].

El problema del flujo de pacientes consiste en establecer las acciones de carácter logístico que se deben tomar en los sistemas que proveen servicios de salud para favorecer la circulación de sus usuarios a través de sus unidades funcionales con el fin de favorecer tanto el uso de recursos (eficiencia) como la oportunidad en la prestación del servicio (eficacia). Típicamente se busca analizar la circulación de pacientes desde que ingresan por urgencias hasta su egreso pasando por hospitalización y recibiendo servicios complementarios como toma de imágenes diagnósticas, pruebas de laboratorio, cirugías, entre otros [18, p. 213].

El análisis de la gestión de la capacidad en hospitales requiere de enfoques que permitan integrar las características intrínsecas de su operación (desempeño como un servicio, flujo de pacientes, gestión de recursos), su impacto en las variables de salida (giro cama, porcentaje ocupacional y estancia promedio) y los parámetros de políticas de mejora (capacidad instalada, eficiencia en el uso del recursos, eficacia en la atención) [19, p. 38]. La Fig. 1 representa la forma como estos tópicos dan pie a la formulación de las preguntas de investigación que se abordan en el presente artículo.

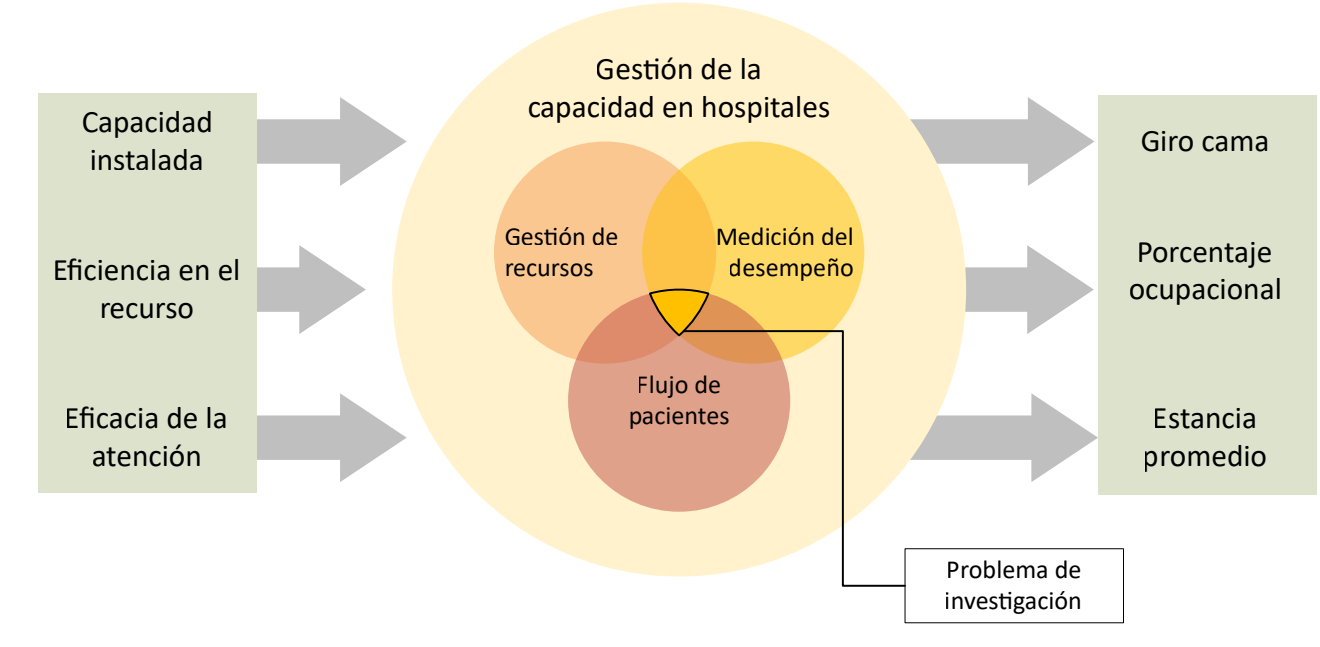

Fig. 1. Problemática de la gestión de capacidades en hospitales. Fuente: Autores.

El desarrollo de la investigación se construyó a partir de los siguientes cuestionamientos:

1. ¿Bajo qué perspectiva de análisis se pueden integrar los tópicos de gestión de recursos, medición del desempeño y flujo de pacientes en instituciones hospitalarias?

2. ¿Cómo impactan las políticas de gestión de capacidad en los indicadores de eficiencia hospitalaria global (giro cama, porcentaje ocupacional y estancia promedio)?

3. ¿Cómo se puede estudiar la evolución en el tiempo que tienen los indicadores de eficiencia hospitalaria global y su relación tanto con variables exógenas (demanda) como endógenas (capacidad disponible)? 
Para abordar estos problemas, el equipo de investigación se decantó por el análisis del problema utilizando la metodología de Dinámica de Sistemas con un estudio clasificado como longitudinal secuencial exploratorio [20]. El elección de la Dinámica de Sistemas se sustenta en que es una de las herramientas más relevantes para el estudio de la complejidad, dado que a partir del uso de técnicas de simulación permite el análisis de flujos de materiales e información, así como el estudio de las demoras en estos flujos [21], [22].

\section{REVISIÓN DE LITERATURA}

Los sistemas hospitalarios se caracterizan por enfrentar una inmensa complejidad en sus operaciones. Klein y Young [23, p. 107] realizan un interesante análisis en el que caracterizan a los sistemas hospitalarios como "hipercomplejos" dado que en ellos confluye un gran número de esferas (industrial, científica, profesional, pública y política), objetivos específicos y sociales simultáneamente, un gran número de partes interesadas en sus actividades, múltiples variables que interactúan en su operación, procesos de retroalimentación variados y finalmente un gran tamaño en comparación con otros sectores económicos.

La complejidad de los sistemas hospitalarios radica en sus características de oferta y demanda. En materia de oferta, las mejoras del desempeño de cada unidad funcional (urgencias, cirugía, hospitalización, etc.) no necesariamente contribuyen al desempeño global del sistema de manera proporcional. Las unidades funcionales interactúan entre sí de tal suerte que la mejora en una de ellas puede generar contratiempos en otras, trayendo consigo una afectación al desempeño global [24]. En cuanto a la complejidad de la demanda, Rohleder et al. [25] establecen que la gran diversidad de pacientes en términos de sus patologías, requerimientos, caracterización fisiológica y tipo de aseguramiento generan un enramado de "rutas" al interior las instituciones hospitalarias a tal punto que se podrían asemejar a sistemas de manufactura tipo job shop, donde cada paciente requiere la atención de distintos servicios en modalidades, tiempos y lugares de difícil previsión.

Para abordar la complejidad de estos sistemas, la literatura destaca el uso de la Dinámica de sistemas, como un enfoque que involucra el desarrollo de modelos de simulación que ilustran procesos de acumulación y retroalimentación cuyos resultados pueden ser probados sistemáticamente para formular políticas adecuadas [26, p. 452]. Al desarrollar modelos de simulación con Dinámica de sistemas, es posible examinar el comportamiento de los sistemas en el mediano y largo plazo como resultado de nuevas políticas implementadas [27]. Los modelos de simulación construidos clasifican sus componentes en parámetros, niveles, flujos y variables auxiliares. Los niveles representan acumulaciones de material o información en un sistema, los flujos permiten que existan entradas o salidas desde los niveles, y las variables auxiliares y parámetros condicionan el comportamiento de los flujos de acuerdo con otros componentes del sistema o con decisiones externas al mismo [28].

En el campo de la administración de servicios de salud, la metodología ha sido utilizada para el estudio de problemas de carácter táctico y estratégico fundamentalmente. Las ventajas de su uso radican en que no se requiere un soporte significativamente detallado en los datos cuantitativos para la construcción del modelos [25], la disminución del efecto de la aleatoriedad en el manejo de las variables y la integración de los elementos, procesos y flujos con una visión sistémica [24]. En el plano estratégico, la metodología permite evaluar el impacto de políticas públicas en el bienestar de la población [18], [26], mientras que en el táctico, se observan aplicaciones como las del análisis del flujo de pacientes dentro de instituciones hospitalarias o sistemas de salud [29], [30].

El uso de la Dinámica de sistemas para analizar flujos de pacientes tiene punto de partida el notable trabajo de Lane et al. [24]. En él, se involucra una conceptualización del flujo de pacientes a través de diagramas causales; la identificación de variables críticas como las camas disponibles y el tiempo de alistamiento; y finalmente el análisis de medidas de desempeño asociadas a tiempos de espera, cancelaciones y niveles de ocupación diaria del hospital. En un posterior trabajo, Vanderby y Carter [31] realizaron un ejercicio en el cual estudian los flujos en las etapas de triage, emergencias, hospitalización y egreso a la luz de variables como la edad del paciente, las demoras para recibir camas, el número de pacientes en espera, y la tipología de servicios brindados tanto en urgencias como en hospitalización. 
La falta de datos es uno de los principales obstáculos en la construcción de modelos basados en dinámica de sistemas para el análisis de flujos de pacientes. Brailsford et al. [32] plantean que estas circunstancias, propias de los sistemas complejos, pueden ser afrontadas a partir de la mezcla de un profundo examen de la estructura cualitativa de los modelos y el uso de procedimientos de validación clásicos cuantitativos orientados hacia el resultado del modelo y no hacia su estructura.

Trabajos más recientes en materia de flujo de pacientes utilizando dinámica de sistemas abordan el tema del estudio de la capacidad en áreas específicas de hospitales como Urgencias [33]-[35] y desde una mirada más estratégica, la reacción de sistemas hospitalarios ante la implementación de políticas de transformación del servicio [36].

\section{MetodoloGía}

Para dar respuesta a la primera pregunta de investigación, el presente trabajo se construyó alrededor de la metodología de la Dinámica de sistemas, realizando adaptaciones que permitieron su aplicación al campo del flujo de pacientes. Se inició con el análisis descriptivo del sistema en estudio detallando sus variables y relaciones de causalidad. Como resultado de ello se construyeron diagramas causales, los cuales permitieron compartir modelos mentales en el equipo de trabajo acerca del funcionamiento del sistema. En segunda fase se construyó un modelo de simulación computacional utilizando diagramas de flujos y niveles (también llamados Diagramas de Forrester). Este modelo fue validado comparando el comportamiento de las tres medidas de eficiencia global con sus respectivos datos históricos. En una fase posterior, se diseñaron y ejecutaron experimentos aplicando cambios en variables de entrada del sistema y midiendo su impacto en las medidas de eficiencia global. Los resultados fueron estudiados estadísticamente y se profundizó en algunos de ellos a través del análisis de componentes principales llegando a identificar aquellos parámetros con mayor incidencia en la eficiencia del sistema.

El objeto de estudio seleccionado para el artículo fue una institución hospitalaria de alta complejidad (nivel III) y de carácter público en Colombia cuya identificación prefirió ser reservada por la entidad dado el carácter estratégico de la información divulgada. La información fue recolectada a partir de los informes de gestión y de entrevistas con personal del hospital y expertos de otras instituciones vinculados al sector salud. Específicamente, la investigación analizó el proceso de flujo de pacientes en la institución, comenzando desde su ingreso a través de tres posibles entradas: urgencias, consulta externa y referencia. El proceso estudiado incluyó las operaciones de traslado de pacientes desde urgencias hacia hospitalización, su permanencia en esta área y su posterior salida tras haber recibido el tratamiento médico respectivo. Frente a la diversidad de pacientes y dada la carencia de datos específicos, el estudio se realizó sin realizar diferenciaciones de cohortes por sexo, patología, edad, nivel de aseguramiento u otras condiciones particulares, acogiendo las recomendaciones que para estos casos determina Gunal [30, p. 23].

A continuación se presentan los pasos desarrollados para la aplicación de la metodología de Dinámica de Sistemas en el objeto de estudio referido. En primer lugar, se presenta la hipótesis dinámica, donde se detallan los componentes principales del modelo y la forma como se relacionan de manera cualitativa. En segunda instancia se construye un modelo de simulación y se introduce la formulación matemática para cada componente del sistema. En una tercera fase se realizó la validación del modelo sometiéndolo a distintas condiciones que aseguraran su fiabilidad. Finalmente se introduce el diseño de políticas y evaluación de resultados. Para obtener un mayor detalle sobre los aspectos teóricos de la metodología, se sugiere consultar [28] y [21].

\section{A. Hipótesis dinámica}

Para esta fase se construyó un diagrama causal que permitiera comprender las relaciones entre las variables que componen el sistema, así como su alcance. El diagrama causal expone la relación entre los tres indicadores básicos de eficiencia hospitalaria con las variables independientes y los parámetros del sistema, tales como los días de estancia por paciente, las camas 
instaladas, el arribo de pacientes y la capacidad resolutiva en el área de urgencias. Para su construcción se desarrollaron grupos focales con personal administrativo y médico de la institución a fin de comprender la estructura de variables y las causalidades del sistema.

Preliminarmente fue necesario detallar la forma como los tres indicadores básicos de eficiencia hospitalaria están relacionados entre sí [14]. Estos indicadores fueron adoptados en el diagrama causal como un mecanismo para evaluar el impacto de las estrategias de gestión de la capacidad (número de camas instaladas, días de estancia por paciente y flujo de pacientes que ingresan a hospitalización) en el desempeño operativo del sistema.

El siguiente diagrama (Fig. 2) presenta la forma como estos tres indicadores (encerrados en círculos) dependen de la forma como se administre la capacidad instalada (camas) y la eficacia en los tratamientos (egresos).

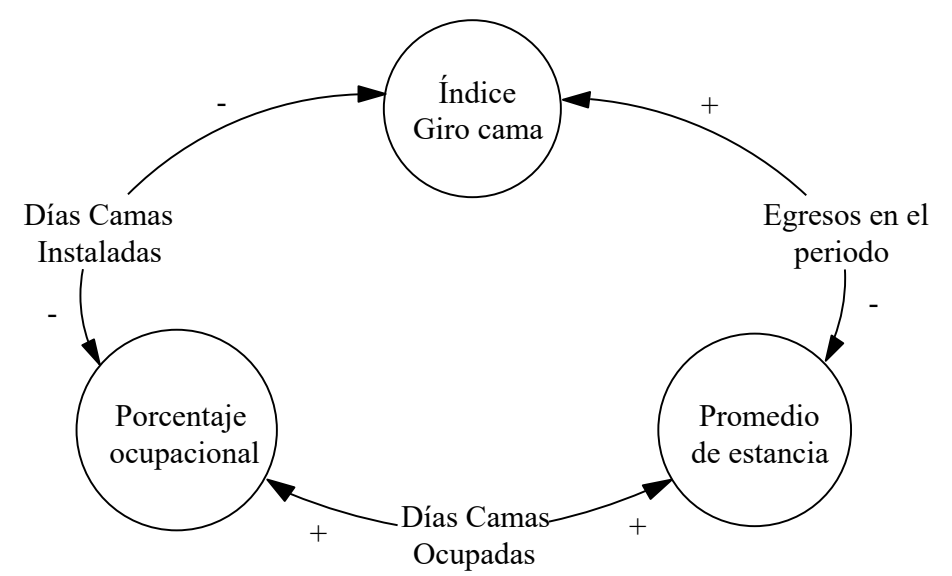

Fig. 2. Relación causal entre parámetros y medidas de desempeño (círculos). Fuente: Autores.

La hipótesis dinámica fue construida alrededor de cuatro niveles específicos (presentados en recuadros en la Fig. 3) que condicionan el flujo de pacientes: Personas en espera en Urgencias, Personas atendidas en Urgencias, Personas en espera de ser Hospitalizadas y Pacientes Hospitalizados. En escenarios reales, la gerencia de una institución hospitalaria busca mantener un equilibrio en los cuatro niveles identificados, de manera que no exista una sobreocupación, pero sin generar un desaprovechamiento de recursos. Para lograr este objetivo, las principales estrategias en el área de hospitalización consisten en incrementar la capacidad del sistema a través de más "Camas instaladas", o mejorar su eficiencia con un control sobre los "Días de estancia”. El diagrama causal completo se presenta en la Fig. 3.

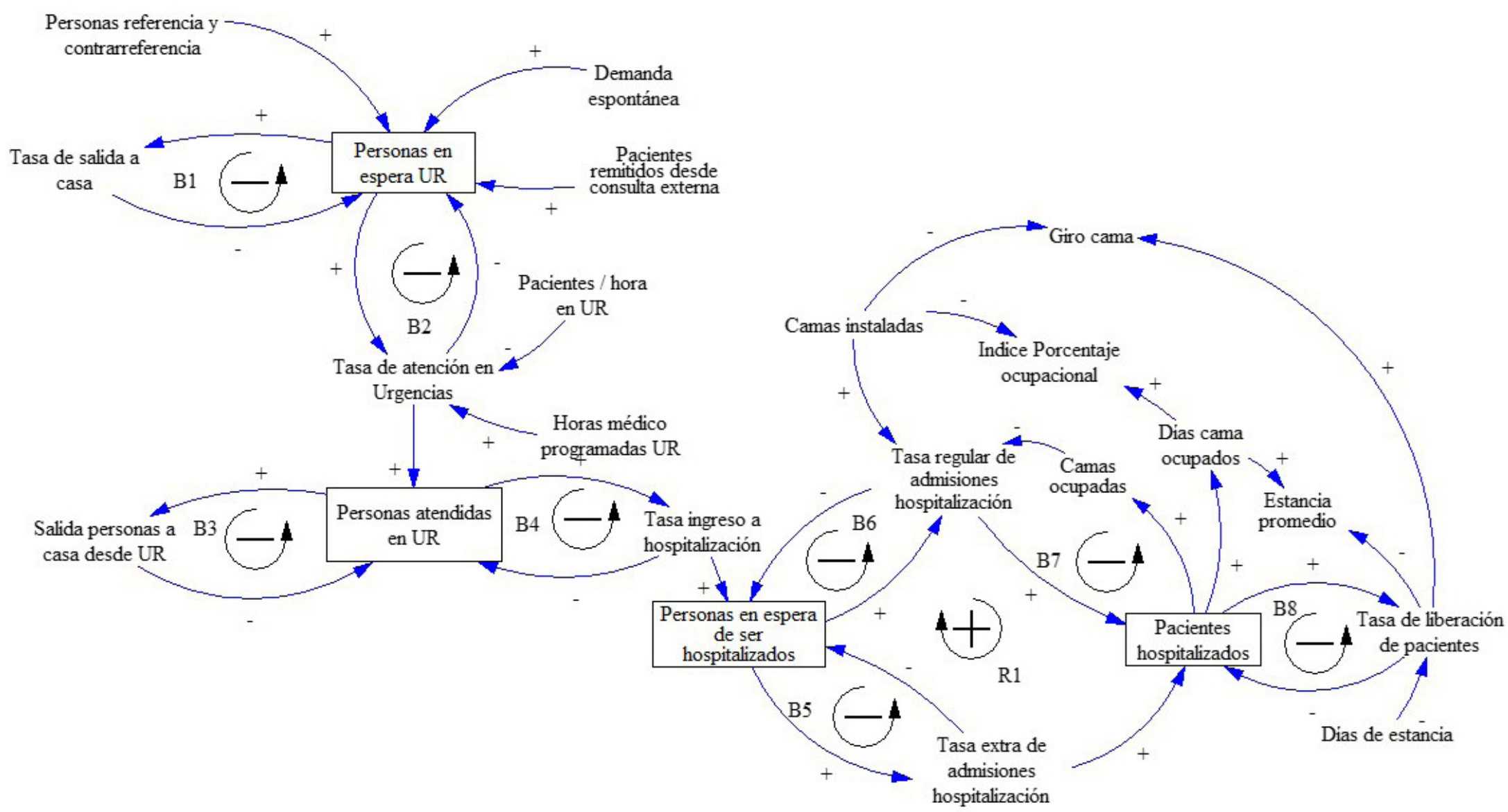

Fig. 3. Diagrama Causal del flujo de pacientes. Fuente: Autores. 
La cantidad de "Personas en espera de atención en urgencias" depende de los arribos por demanda espontánea, referencia y contrarreferencia y consulta externa. También depende del número de personas que abandonan el sistema sin ser atendidas (Ciclo B1 y variables conexas). Tras ser atendidas, estas personas pasan a otro estadio (nivel) que se denota como "Personas atendidas en urgencias" (Ciclo B2). Su incremento impacta proporcionalmente en la remisión de personas a sus hogares (Ciclo B3) y especialmente en la transferencia hacia hospitalización (Ciclo B4).

Antes de ser hospitalizadas, las personas deben esperar protocolos de ingreso, lo cual implica una demora representada por el nivel "Personas en espera de ser hospitalizados". Esta es la condición en la cual un paciente ha sido remitido para que se le asigne una habitación en hospitalización, pero al no haber capacidad suficiente (diferencia entre camas instaladas y camas ocupadas), deberá esperar en un área intermedia. Existen dos rutas para que el paciente pueda finalmente ser hospitalizado, y ello se representa por la "Tasa regular de admisiones en hospitalización" (Ciclos B6 y B7) y por la "Tasa extra de admisiones en hospitalización" (Ciclo B5). Esta última se refiere a aquellos pacientes que deben ser ubicados en áreas que son adicionadas extraoficialmente a la capacidad de hospitalización (pabellones de otros servicios distintos). En la medida en que las tasas de hospitalización aumenten, se incrementa el nivel de "Pacientes hospitalizados". Este nivel se disminuye por la acción de la "Tasa de liberación de pacientes" (Ciclo B8).

Mientras que los ciclos identificados hasta ahora (B1 hasta B8) son todos de balance, el ciclo $\mathrm{R} 1$ consiste en un ciclo de refuerzo que promueve la eficiencia del sistema. A continuación, su explicación detallada: Un aumento en las "Camas instaladas" del hospital permite un incremento en la "Tasa regular de admisiones". Con esta mejora, es de esperarse que se disminuya la cantidad de "Personas en espera de ser hospitalizados" y por lo tanto también disminuirá la "Tasa extra de admisiones". Ello quiere decir que menos personas estarán presionando para ser ingresadas a hospitalización. Como hay una reducción en el crecimiento de "Pacientes hospitalizados", se lograrán liberar más camas, es decir que también disminuye el crecimiento de la variable de "Camas ocupadas" y como consecuencia se dará un incremento en la Tasa regular de admisiones.

La condición positiva del ciclo refuerza la estrategia de que el aumento de camas instaladas mejoraría la eficiencia global del sistema. No obstante, este ciclo de refuerzo y sus niveles de "Personas en espera de ser hospitalizados" y "Pacientes hospitalizados" se equilibran con ciclos de balance como el B4 y el B8: Mientras que el aumento de "Camas instaladas", reduce el crecimiento del nivel de "Pacientes hospitalizados", el aumento en los "Días de estancia" produce un efecto contrario, reforzando su crecimiento. Igual comportamiento se advierte con la influencia de la "Tasa de ingreso a hospitalización" y el nivel de "Personas en espera de ser hospitalizadas".

La construcción de la hipótesis dinámica permitió comprender la forma como los indicadores de desempeño hospitalarios se ven influenciados por las políticas de aumento de la capacidad (camas instaladas y tasa ingreso a hospitalización), y de la administración del recurso (días de estancia). Las relaciones de causalidad se resumen en la Tabla 1.

TABla 1. COMPORTAMIENTO DE LOS INDICADORES DE EFICIENCIA HOSPITALARIA FRENTE A AFECTACIONES DE LA CAPACIDAD.

\begin{tabular}{|c|c|c|c|c|c|}
\hline \multirow{3}{*}{$\begin{array}{l}\text { Parámetro afectado } \\
\text { Camas instaladas }\end{array}$} & \multirow{3}{*}{$\begin{array}{l}\begin{array}{l}\text { Política aplicada } \\
\text { sobre parámetro }\end{array} \\
(+)\end{array}$} & \multicolumn{4}{|c|}{ Respuesta de los indicadores de eficiencia hospitalaria } \\
\hline & & \multicolumn{2}{|c|}{ Giro cama } & Porcentaje ocupacional & \multirow[t]{2}{*}{ Estancia promedio } \\
\hline & & $(-)$ & $(-)$ & Relativo & \\
\hline Camas instaladas & $(-)$ & $(+)$ & $(+)$ & Relativo & \\
\hline Días de estancia & $(+)$ & $(-)$ & $(+)$ & $(+)$ & \\
\hline Días de estancia & $(-)$ & $(+)$ & $(-)$ & $(-)$ & \\
\hline Tasa ingreso hospitalización & $(+)$ & $(+)$ & $(+)$ & Relativo & \\
\hline Tasa ingreso hospitalización & $(-)$ & $(-)$ & $(-)$ & Relativo & \\
\hline
\end{tabular}

Fuente: Autores. 
Tres políticas de mejora utilizadas en la gerencia hospitalaria consisten en el aumento del número de camas instaladas, aumento del flujo de pacientes de urgencias a hospitalización y la disminución de los días de estancia. La aplicación de estas políticas en simultánea puede generar resultados que dependen de su magnitud. Por ejemplo, un aumento en la tasa de ingreso a hospitalización aumenta el porcentaje ocupacional, pero un aumento en las camas instaladas tiende a disminuir este porcentaje. La estancia promedio depende de otros factores y de la magnitud de la afectación en los parámetros, razón por la cual se califica su respuesta como "relativa".

En consecuencia, es necesario plantear un modelamiento cuantitativo que permita examinar el resultado de la aplicación de estas políticas teniendo en cuenta su magnitud, así como el impacto de su combinación en asocio con factores como los crecimientos de demanda. Para estudiar con detalle estas relaciones se pasó a la siguiente fase de elaboración de un modelo de simulación computacional.

\section{B. Modelo de simulación computacional}

Un modelo computacional fue construido con el fin de realizar la simulación del sistema. Para ello se utilizaron Diagramas de Forrester en el aplicativo Vensim 6.3 DSS. El modelo construido representa el flujo de personas que ingresan a un hospital y su circulación a través del mismo hasta que salen del sistema (Fig. 4). En el primer nivel "Pacientes Espera Urgencias" (PEU) se registran los arribos de pacientes al sistema por referencia de otras instituciones (TIR), ingreso por consulta externa (TIC), y demanda espontánea (TIDU). También se consideró la posibilidad de que los pacientes se retiren de este nivel a partir del flujo "Tasa Deserción Urgencias" (TDU).

La "Tasa de Servicio de Urgencias" (TSU) conecta el nivel anterior con el nivel de "Pacientes atención Urgencias" (PAU). Este flujo depende a su vez de parámetros de productividad como las "Horas Médico Diarias" (HMD) y el número de "Pacientes atendidos por Hora Médico" (PHM). El nivel de "Pacientes Atención Urgencias" (PAU) permite tanto la salida de pacientes del sistema "Tasa Alta Urgencias" (TAU) como su paso a hospitalización a partir del flujo "Tasa Ingreso Hospitalización” (TIH).

En un escenario real es común encontrar que exista sobreocupación del área de hospitalización en algunos días o temporadas del año. Para modelar esto, se construyeron dos flujos que se desprenden del nivel "Pacientes Espera Hospitalización” (PEH). El flujo superior implica que un porcentaje de estos pacientes fueron hospitalizados en áreas que hacen parte de su capacidad regular a una velocidad regulada por la "Tasa Regular de Admisiones Hospitalización” (TRAH). Otro porcentaje de pacientes que ingresan a hospitalización deben ser ubicados en áreas que inicialmente no están acondicionadas para ello (salas de otras especialidades o habitaciones sobreocupadas). Este fenómeno se denominó "Hospitalización en Expansión" y está modelado por el flujo inferior que se desprende del nivel "Pacientes Espera Hospitalización" (PEH). Este flujo está condicionado por la "Tasa Admisiones Expansión Hospitalización” (TAEH). Los dos flujos (regular y en expansión) conllevan al nivel de "Pacientes Hospitalizados" (PHO). Tras recibir su tratamiento, los "Pacientes Hospitalizados" (PHO) se convierten en "Pacientes Egresados" (PEG) debido al flujo regulado por la "Tasa de Egresos de Pacientes" (TEP).

Los indicadores de eficiencia hospitalaria global fueron incluidos en el modelo (Fig. 4) y se destacan por tener un recuadro con fondo gris. El "Giro cama mensual promedio" (GCMP) depende de la capacidad instalada ("Camas Instaladas" (CDIS)) y el número de pacientes que pasan por ellas en una franja de tiempo "Tasa de Egresos de Pacientes" (TEP). El "Porcentaje Ocupacional Mensual" (POM) fue modelado como una relación entre los "Días Cama Ocupados" (DCO) y los "Días Cama Disponibles por Día" (DCDD). Finalmente, la "Estancia Promedio" (EPM) se calculó como el cociente del número de "Días Cama Ocupados" (DCO) y el total de "Pacientes Egresados" (PEG) [Ver ecuaciones (1), (2) y (3)]. Para esta construcción fue necesario incluir un flujo adicional que permitiera calcular el nivel de "Días Cama Ocupados" (DCO). Este nivel es de carácter abstracto y se utilizó para definir la variable acumulada de días cama que relaciona las variables POM y EPM. 


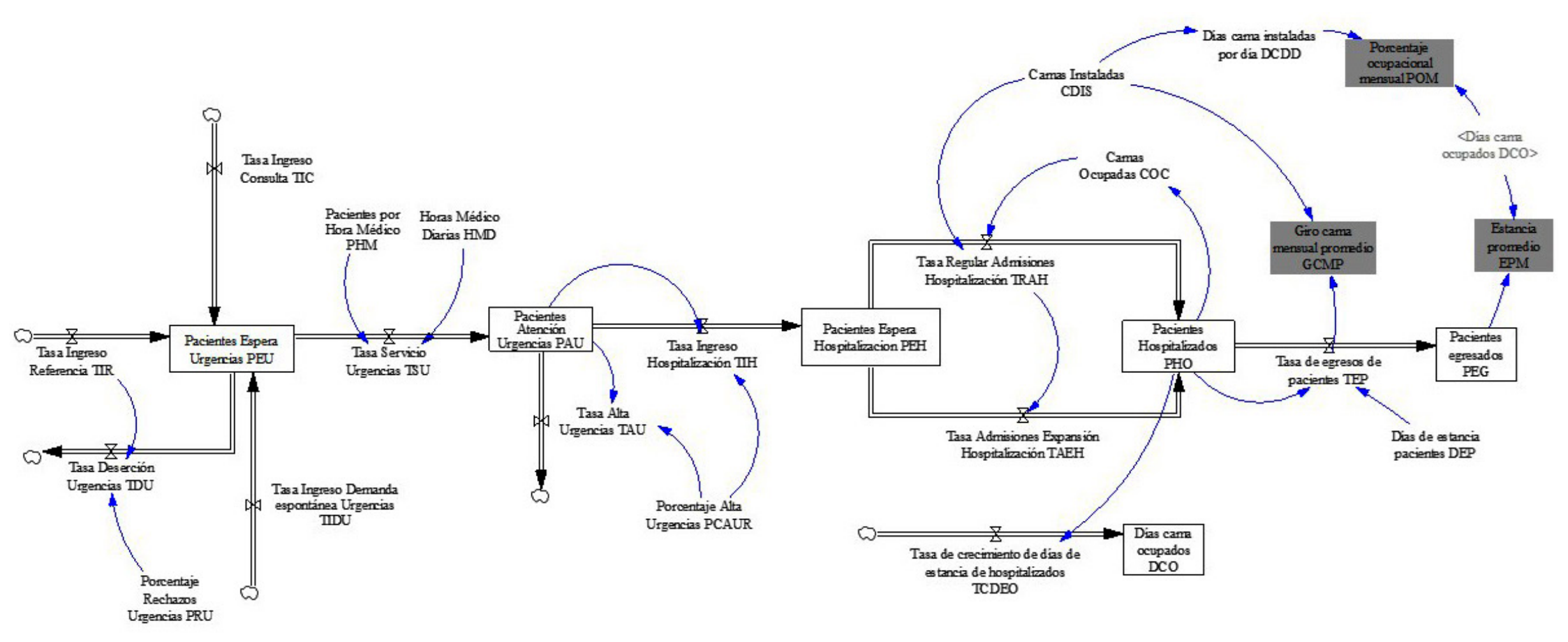

Fig. 4. Diagrama de Forrester para el flujo de pacientes (Versión resumida). Fuente: Autores.

La estructura del modelo puede ser estudiada desde dos puntos de vista: técnico y funcional. En el plano técnico se busca analizar el modelo desde la teoría de modelamiento de Dinámica de Sistemas. Los componentes del modelo se clasifican en variables, niveles y flujos. Los niveles son acumuladores de material, información o dimensiones abstractas. Los flujos afectan a los niveles aumentándoles o disminuyéndoles a lo largo del tiempo. Las variables permiten la conexión entre flujos y niveles para efectos de cálculos matemáticos. Para una comprensión detallada del significado de estos elementos se recomienda revisar a Sterman [28, p. 191].

Desde el punto de vista funcional, el modelo de simulación está compuesto por parámetros, variables independientes, medidas de desempeño y variables auxiliares. Los parámetros representan las políticas o estrategias implementadas sobre el sistema como variables de entrada. Las variables independientes se consideran exógenas al modelo y están gobernadas por factores no controlables, pero sí medibles.

Las medidas de desempeño permiten comprender el estado del sistema dado que generan valores de salida que representan la consecuencia de la interacción entre variables independientes, parámetros y variables auxiliares. Finalmente, las variables auxiliares sirven de conectoras entre valores de entrada y de salida del modelo.

Para examinar el detalle de la naturaleza técnica y funcional del modelo construido, se presenta a continuación el detalle de los principales componentes del modelo. Se utilizó para ello la guía de reporte propuesta por Rahmandad y Sterman [37] para la descripción sistemática de modelos basados en Dinámica de Sistemas (Tabla 2).

Tabla 2. Principales componentes del modelo matemático de Flujo de Pacientes DEtallando SU FORMULACIÓN, DESCRIPCIÓN, UNIDADES, CARÁCTER TÉCNICO Y FUNCIONAL.

\begin{tabular}{|c|c|c|c|}
\hline Fórmulas y comentarios & Unidades & $\begin{array}{l}\text { Carácter } \\
\text { técnico }\end{array}$ & $\begin{array}{l}\text { Carácter } \\
\text { funcional }\end{array}$ \\
\hline $\begin{array}{l}\mathrm{PCU}(\mathrm{t})=\mathrm{PCU}(0)+\int_{0} \mathrm{t}(\mathrm{TIC}(\mathrm{s})+\mathrm{TIR}(\mathrm{s})+\mathrm{TIDU}(\mathrm{s})-\mathrm{PRU}(\mathrm{s})-\mathrm{TSU}(\mathrm{s})) \mathrm{ds}(4) \\
\text { El nivel de pacientes en espera en urgencias }(\mathrm{PCU}) \text { se incrementa en función de } \\
\text { nuevos pacientes que ingresan desde consulta externa (TIC), referencia (TIR) o } \\
\text { demanda espontánea (TIDU). El nivel disminuye por pacientes que ingresan a } \\
\text { ser atendidos (TSU) o que desertan del servicio de urgencias (TDU). }\end{array}$ & Personas & Nivel & $\begin{array}{l}\text { Medida de } \\
\text { desempeño }\end{array}$ \\
\hline $\begin{array}{l}\text { PAU }(\mathrm{t})=\mathrm{PAU}(0)+\int_{0} \mathrm{t}(\mathrm{TSU}(\mathrm{s})-\mathrm{TAU}(\mathrm{s})-\mathrm{TIH}(\mathrm{s})) \mathrm{ds}(5) \\
\text { El nivel de pacientes que reciben atención en urgencias (PAU) crece según lo } \\
\text { hace el flujo de pacientes que ingresan a ser atendidos (TSU) pero también } \\
\text { disminuye por efectos de aquellos que son dados de alta (TAU) o que pasan a } \\
\text { ser hospitalizados (TIH). }\end{array}$ & Personas & Nivel & $\begin{array}{l}\text { Medida de } \\
\text { desempeño }\end{array}$ \\
\hline
\end{tabular}




\begin{tabular}{|c|c|c|c|}
\hline Fórmulas y comentarios & Unidades & $\begin{array}{l}\text { Carácter } \\
\text { técnico }\end{array}$ & $\begin{array}{l}\text { Carácter } \\
\text { funcional }\end{array}$ \\
\hline $\begin{array}{l}\mathrm{PEH}(\mathrm{t})=\mathrm{PEH}(0)+\int_{0} \mathrm{t}(\mathrm{TIH}(\mathrm{s})-\mathrm{TRAH}(\mathrm{s})-\mathrm{TAEH}(\mathrm{s})) \mathrm{ds}(6) \\
\text { El número de pacientes en espera de ser hospitalizados }(\mathrm{PEH}) \text { depende de la } \\
\text { tasa de ingresos a hospitalización }(\mathrm{TIH}) \text { y de dos tasas que le disminuyen. } \\
\text { En primer lugar, la tasa regular de admisiones a hospitalización (TRAH) } \\
\text { y en segundo lugar la tasa de admisiones en expansión a hospitalización } \\
\text { (TAEH). Esta última refiere a aquellos pacientes que son ubicados en áreas } \\
\text { originalmente no diseñadas para alojar pacientes. }\end{array}$ & Personas & Nivel & $\begin{array}{l}\text { Medida de } \\
\text { desempeño }\end{array}$ \\
\hline $\begin{array}{l}\mathrm{PHO}(\mathrm{t})=\mathrm{PHO}(0)+\int_{0}^{\mathrm{t}}(\mathrm{TRAH}(\mathrm{s})+\mathrm{TAEH}(\mathrm{s})-\mathrm{TEP}(\mathrm{s})) \mathrm{ds}(7) \\
\text { El aumento en las tasas de admisiones de manera regular }(\mathrm{TRAH}) \mathrm{y} \\
\text { en expansión }(\mathrm{TAEH}) \text { hace que se incremente el número de pacientes } \\
\text { hospitalizados }(\mathrm{PHO}) \text {. Este último valor se ve reducido por la tasa de egresos de } \\
\text { pacientes (TEP) es decir aquellos que son dados de alta. }\end{array}$ & Personas & Nivel & $\begin{array}{l}\text { Medida de } \\
\text { desempeño }\end{array}$ \\
\hline $\begin{array}{l}\text { PEG(t) = PEG(0) }+\int_{0} \mathrm{t} T E P(s) d s(8) \\
\text { El nivel de pacientes egresados }(P E G) \text { crece en la medida que avanza el } \\
\text { tiempo del proceso y esto se da por el flujo de pacientes que egresan desde } \\
\text { hospitalización }(T E P) \text {. }\end{array}$ & Personas & Nivel & $\begin{array}{l}\text { Medida de } \\
\text { desempeño }\end{array}$ \\
\hline $\begin{array}{l}T S U(t)=\min \left\{\begin{array}{c}P H M(t) * H M D(t) \\
M a x O u t U r\end{array}\right. \\
\text { La tasa de servicio en urgencias (TSU) es una función que calcula el flujo de } \\
\text { pacientes que se da desde la sala de espera hasta los consultorios de urgencias. } \\
\text { Consiste en el mínimo de dos valores: el producto del número de horas médico } \\
\text { disponibles (HMD) y el número de pacientes que son atendidos en una hora } \\
\text { médico (PHM); y por otro lado el número de pacientes que están esperando } \\
\text { servicio de urgencias (MaxOutUr) }\end{array}$ & $\begin{array}{l}\text { Personas / } \\
\text { día }\end{array}$ & Flujo & $\begin{array}{l}\text { Variable } \\
\text { auxiliar }\end{array}$ \\
\hline $\begin{array}{l}\mathrm{TIH}(\mathrm{t})=\mathrm{PAU}(\mathrm{t}) *(1-\mathrm{PCAUR}(\mathrm{t}))(10) \\
\text { La tasa de ingreso a hospitalización (TIH) refleja el flujo de personas } \\
\text { que pasan de haber sido atendidas en urgencias a requerir el ingreso a } \\
\text { hospitalización. Se calcula como el producto del nivel de pacientes recibiendo } \\
\text { atención en urgencias (PAU) y el inverso aditivo del porcentaje de pacientes que } \\
\text { son dados de alta desde urgencias (PCAUR). }\end{array}$ & $\begin{array}{l}\text { Personas / } \\
\text { día }\end{array}$ & Flujo & $\begin{array}{l}\text { Variable } \\
\text { auxiliar }\end{array}$ \\
\hline $\begin{array}{l}\operatorname{TRAH}(t)=\min \left\{\begin{array}{c}\operatorname{CDIS}(t)-\operatorname{COC}(t) \\
\operatorname{MaxOut}(t)\end{array}\right. \\
\text { La tasa regular de admisiones en hospitalización (TRAH) representa el flujo } \\
\text { de personas que son hospitalizadas en condiciones normales, sin ocupar áreas } \\
\text { habilitadas para otros servicios. Es el menor valor entre las camas desocupadas } \\
\text { y una fracción del número de pacientes que están en espera (MaxOut). }\end{array}$ & $\begin{array}{l}\text { Personas / } \\
\text { día }\end{array}$ & Flujo & $\begin{array}{l}\text { Variable } \\
\text { auxiliar }\end{array}$ \\
\hline $\begin{array}{l}\text { TAEH }(t)=\max \left\{\begin{array}{c}0 \\
\text { MaxOut }(t)-T R A H(t)\end{array}\right. \\
\text { Los pacientes que no pueden ingresar deberán ser hospitalizados en áreas } \\
\text { agregadas a este servicio. Su flujo se denomina Tasa de admisiones en } \\
\text { expansión (TAEH) y se calcula como la diferencia entre la fracción de pacientes } \\
\text { en espera para ingresar a hospitalización (MaxOut) y la tasa regular de } \\
\text { admisiones en hospitalización (TRAH). }\end{array}$ & $\begin{array}{l}\text { Personas / } \\
\text { día }\end{array}$ & Flujo & $\begin{array}{l}\text { Variable } \\
\text { auxiliar }\end{array}$ \\
\hline $\begin{array}{l}\text { TEP }(\mathrm{t})=\mathrm{PHO}(\mathrm{t}) / \mathrm{DEP}(\mathrm{t}) * \mathrm{PLP}(\mathrm{t})(13) \\
\text { La tasa de egresos de pacientes (TEP) es un flujo que extrae pacientes del } \\
\text { área de hospitalización. Su cálculo se da como el cociente del nivel de pacientes } \\
\text { hospitalizados }(\mathrm{PHO}) \text { y los días de estancia de pacientes (DEP). }\end{array}$ & $\begin{array}{l}\text { Personas / } \\
\text { día }\end{array}$ & Flujo & $\begin{array}{l}\text { Variable } \\
\text { auxiliar }\end{array}$ \\
\hline $\begin{array}{l}\text { GCMP }(\mathrm{t})=(\mathrm{TEP}(\mathrm{t}) * 30 \text { día/mes }) / \mathrm{CDIS}(\mathrm{t})(14) \\
\text { El giro cama mensual promedio calcula el número de veces que una cama } \\
\text { es ocupada por un paciente distinto en un mes. Se calcula como el cociente } \\
\text { del número de pacientes que son egresados por mes }(\mathrm{TEP} * 30) \text { y las camas } \\
\text { disponibles del hospital (CDIS). }\end{array}$ & $\begin{array}{l}\text { Persona / } \\
\text { (cama * } \\
\text { mes) }\end{array}$ & Variable & $\begin{array}{l}\text { Medida de } \\
\text { desempeño }\end{array}$ \\
\hline $\begin{array}{l}\mathrm{POM}(\mathrm{t})=\mathrm{DCO}(\mathrm{t}) / \mathrm{DCDD}(\mathrm{t})(15) \\
\text { El porcentaje ocupacional mensual }(\mathrm{POM}) \text { se define como el cociente de los } \\
\text { días cama ocupados (DCO) y los días cama disponibles en un mismo periodo } \\
\text { mensual (DCDD). }\end{array}$ & Porcentaje & Variable & $\begin{array}{l}\text { Medida de } \\
\text { desempeño }\end{array}$ \\
\hline
\end{tabular}




\begin{tabular}{|c|c|c|c|}
\hline Fórmulas y comentarios & Unidades & $\begin{array}{l}\text { Carácter } \\
\text { técnico }\end{array}$ & $\begin{array}{l}\text { Carácter } \\
\text { funcional }\end{array}$ \\
\hline $\begin{array}{l}\operatorname{EPM}(\mathrm{t})=\mathrm{DCO}(\mathrm{t}) / \mathrm{PEG}(\mathrm{t})(16) \\
\text { El indicador de Estancia promedio (EPM) relaciona los días cama ocupados } \\
\text { (DCO) y el número de pacientes egresados (PEG) para un mismo periodo de } \\
\text { tiempo. }\end{array}$ & $\begin{array}{l}\text { Días / } \\
\text { persona }\end{array}$ & Variable & $\begin{array}{l}\text { Medida de } \\
\text { desempeño }\end{array}$ \\
\hline $\begin{array}{l}\mathrm{DCO}(\mathrm{t})=\mathrm{DCO}(0)+\int_{0}^{\mathrm{t}}(\mathrm{TCDEO}(\mathrm{s}) \mathrm{ds}(17) \\
\text { Los días cama ocupados (DCO) son calculados como un acumulado del número } \\
\text { de días en que cada cama ha sido ocupada. Por lo tanto, se formula como un } \\
\text { nivel que se incrementa con una tasa de crecimiento (TCDEO) que a su vez } \\
\text { consiste en el número de pacientes hospitalizados (PHO) pasados a días cama } \\
\text { (TCDEO). }\end{array}$ & Días & Variable & Auxiliar \\
\hline $\begin{array}{l}\mathrm{HMD}=69,13(18) \\
\text { Corresponde al número de horas médico-diarias asignadas por la } \\
\text { administración de la institución para atender en Urgencias. }\end{array}$ & Horas/Día & Variable & Parámetro \\
\hline $\begin{array}{l}\text { CDIS = } 210 \text { (19) } \\
\text { Representa el número de camas disponibles instaladas en el área de } \\
\text { hospitalización. }\end{array}$ & $\begin{array}{l}\text { Camas / } \\
\text { Día }\end{array}$ & Variable & Parámetro \\
\hline $\begin{array}{l}\text { CDEP }=8,8(20) \\
\text { El número de días de estancia en hospitalización en promedio para cada } \\
\text { paciente. }\end{array}$ & $\begin{array}{l}\text { Días / } \\
\text { Paciente }\end{array}$ & Variable & Parámetro \\
\hline $\begin{array}{l}\text { TIDU }=23,54(21) \\
\text { El número de días de estancia en hospitalización en promedio para cada } \\
\text { paciente. }\end{array}$ & $\begin{array}{l}\text { Paciente / } \\
\text { Día }\end{array}$ & Flujo & $\begin{array}{l}\text { Variable } \\
\text { indep. }\end{array}$ \\
\hline
\end{tabular}

Fuente: Autores.

\section{Validación del modelo}

La validación del modelo se desarrolló con el apoyo de grupos focales con funcionarios de la institución hospitalaria quienes aprobaron su estructura lógica. Se realizaron corridas de simulación para ajustar el comportamiento de las medidas de desempeño a los resultados históricos evidenciados durante dos años en la institución hospitalaria. La validación fue complementada con el cálculo de coeficientes de variación para cada medida de desempeño y las desviaciones medias absoluta s (Tabla 3).

TABla 3. Resultados DE DATOS HistóRICOS Y MODElO SIMUlAdo PARA MEDIDAS DE DESEMPEÑO DEL SISTEMA (2013-2014).

\begin{tabular}{|l|l|l|l|l|l|l|}
\hline & \multicolumn{2}{|c|}{$\begin{array}{c}\text { EPM } \\
\text { (Días/Paciente) }\end{array}$} & \multicolumn{2}{c|}{$\begin{array}{c}\text { GCMP } \\
\text { (Pacientes / Cama) }\end{array}$} & \multicolumn{2}{c|}{$\begin{array}{c}\text { POM } \\
\text { (Porcentaje) }\end{array}$} \\
\hline & Sim. & His. & Sim. & His. & Sim. & His. \\
\hline Media & 8,80 & 8,67 & 3,81 & 3,78 & 97,66 & 101,43 \\
\hline DE & 0,27 & 0,41 & 0,27 & 0,21 & 1,62 & 10,24 \\
\hline CV & 0,03 & 0,05 & 0,07 & 0,06 & 0,02 & 0,10 \\
\hline DMA & 0,16 & 0,18 & 0,01 & \\
\hline $\begin{array}{l}\text { DE.: Desviación estándar } \\
\text { CV: Coeficiente de variación } \\
\text { DMA: Desviación media absoluta }\end{array}$ \\
\hline
\end{tabular}

Fuente: Autores.

Adicionalmente se realizaron pruebas para evaluar el comportamiento del modelo en condiciones extremas, y de sensibilidad de resultados (Fig. 5 y Fig. 6) [38]. Las conclusiones afirman que el modelo cuenta con plena validez para representar el sistema objeto de estudio y en tal sentido se procedió a desarrollar el proceso de experimentación. 


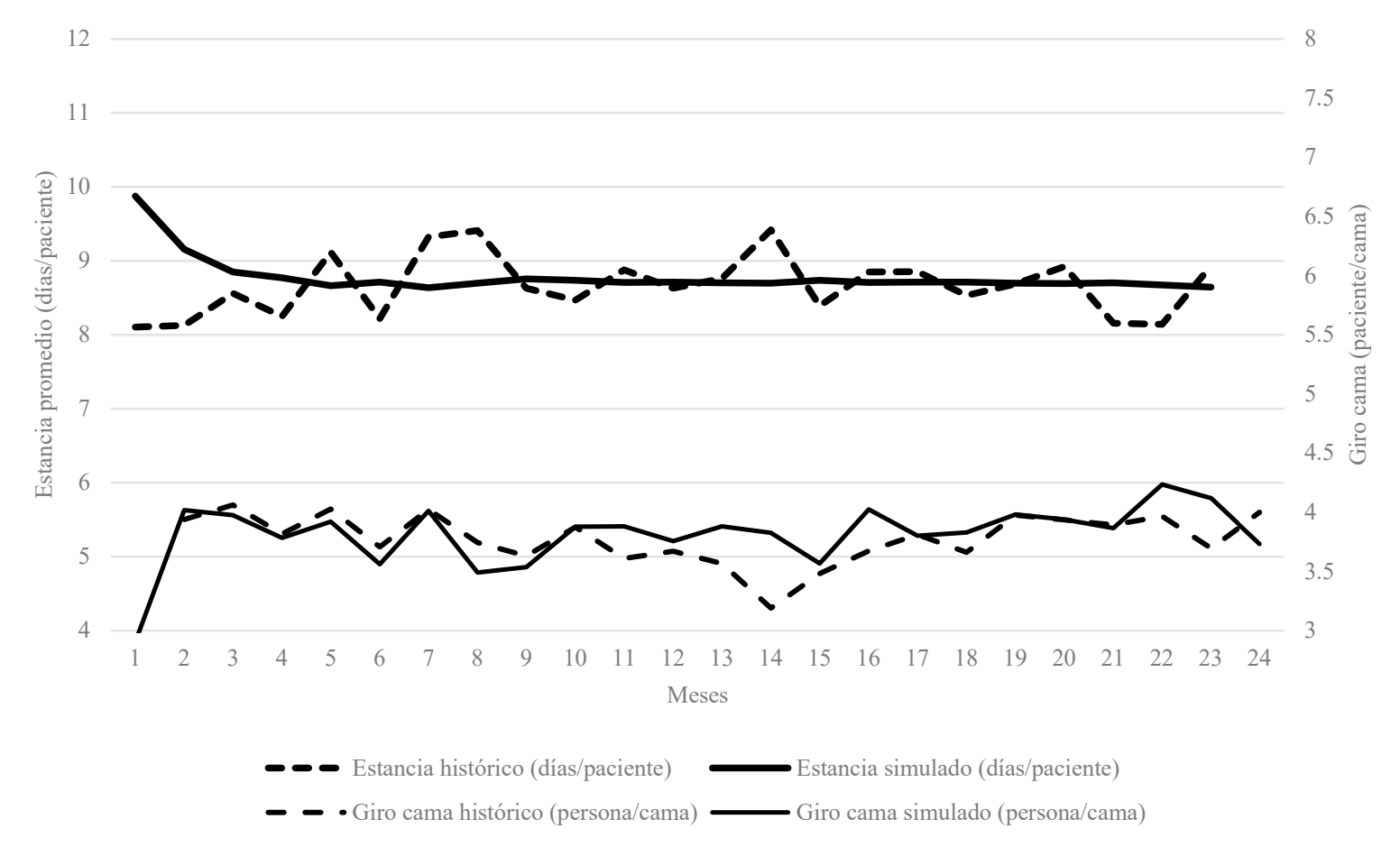

Fig. 5. Giro cama mensual y estancia promedio mensual (histórico y simulado). Fuente: Autores.

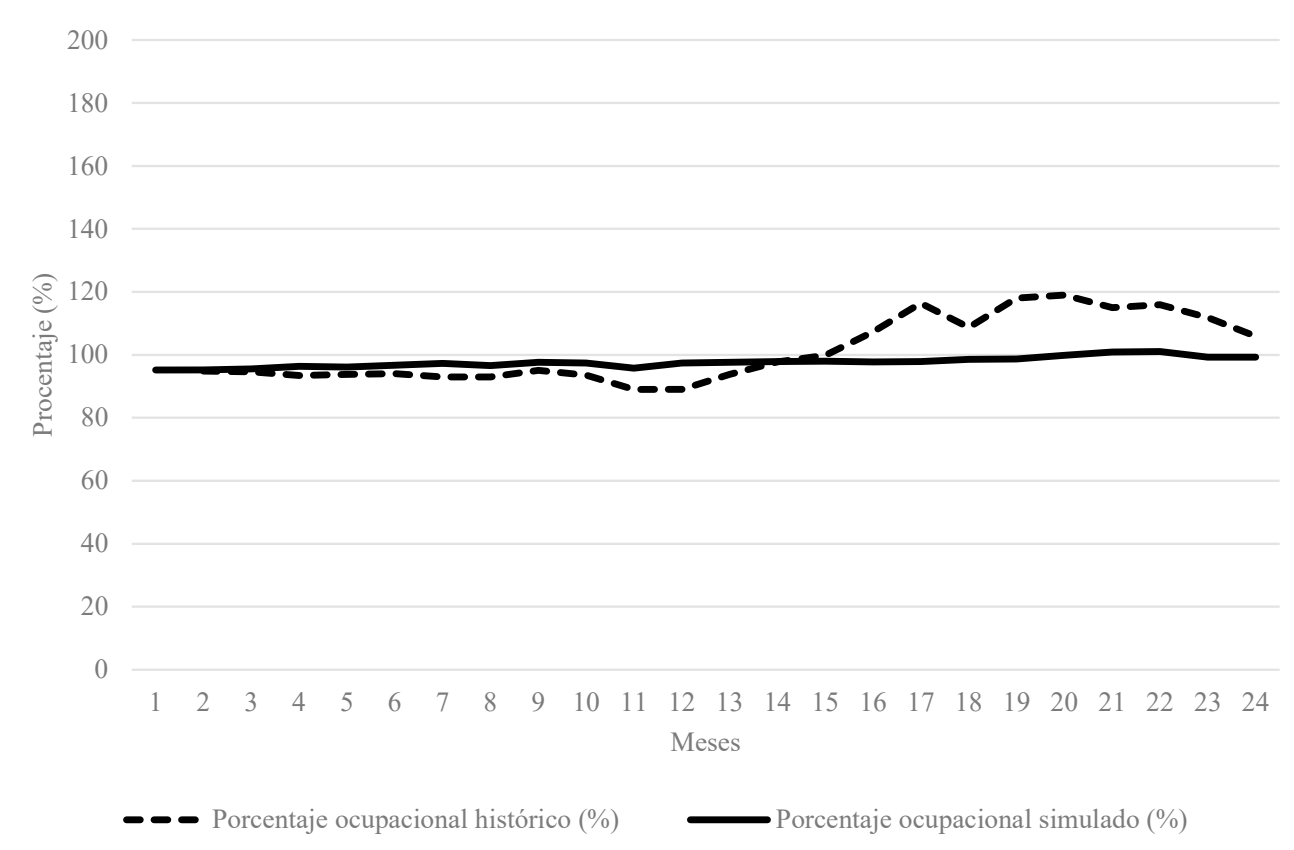

Fig. 6. Porcentaje ocupacional mensual (histórico y simulado). Fuente: Autores.

\section{Análisis de resultados obtenidos}

Con el fin de dar respuesta a la segunda y tercera pregunta de investigación se planteó la necesidad de experimentar con los tres principales parámetros del sistema identificados en la Tabla 1: Días de Estancia de Pacientes (DEP), Horas Médico Diarias (HMD) y Camas Instaladas (CDIS). Las horas médico diarias fueron incluidas como una forma de medir la velocidad con la cual ingresan las personas a hospitalización.

Para la descripción del experimento se utilizará el término "factor" para denotar a cada uno de los parámetros utilizados. En este sentido, el experimento constó de tres factores y cada uno de ellos en dos niveles. Los niveles de los factores fueron categorizados como "Alto" (+) o "Bajo" (-) y sus valores fueron seleccionados por el grupo de trabajo en concordancia con resultados históricos y justificaciones propias de la institución objeto de estudio (Tabla 4).

TABLA 4. VALORES DE LOS Niveles Utilizados PARA CADA FACTOR.

\begin{tabular}{|l|l|l|l|}
\hline & \multicolumn{3}{|c|}{ Factores utilizados } \\
\hline Nivel & DEP & HMD & CDIS \\
\hline Alto $(+)$ & 5 & 89,133333 & 270 \\
\hline Bajo $(-)$ & 8,8 & 69,133333 & 230 \\
\hline
\end{tabular}

Fuente: Autores. 
Los valores correspondientes al nivel "Bajo (-)" obedecen a los promedios que se observaron en la recolección de datos históricos del hospital en estudio. Los valores del nivel "Alto (+)" representan metas establecidas por la gerencia de la institución hospitalaria para la implementación de políticas de mejora. A continuación se explica cada nivel en cada factor.

Para el factor DEP, el nivel Alto obedece a la mejora esperada por la implementación de metodologías como Lean Healthcare en las principales unidades de servicios. Con ello, se espera lograr una reducción de los tiempos de estancia de casi cuatro días representados en trámites para el ingreso y descarga del paciente del área de hospitalización. En el caso del factor HMD, el nivel Alto consiste en la mejora esperada por la contratación de 20 horas médico diarias adicionales. Finalmente, la gestión de camas a través del factor CDIS se puede ver mejorada con la adquisición de 40 nuevas unidades que representan un aumento de la capacidad para el área de hospitalización del 17\%.

Los cambios en cada factor mencionado en la Tabla 4 se aplicaron simulando su implementación de manera gradual desde el día 100 hasta el día 200 de cada corrida (periodo de transición). En la Fig. 7 se evidencia cómo cambiaron los niveles desde un nivel bajo (-) a un nivel alto (+) durante el periodo de transición.

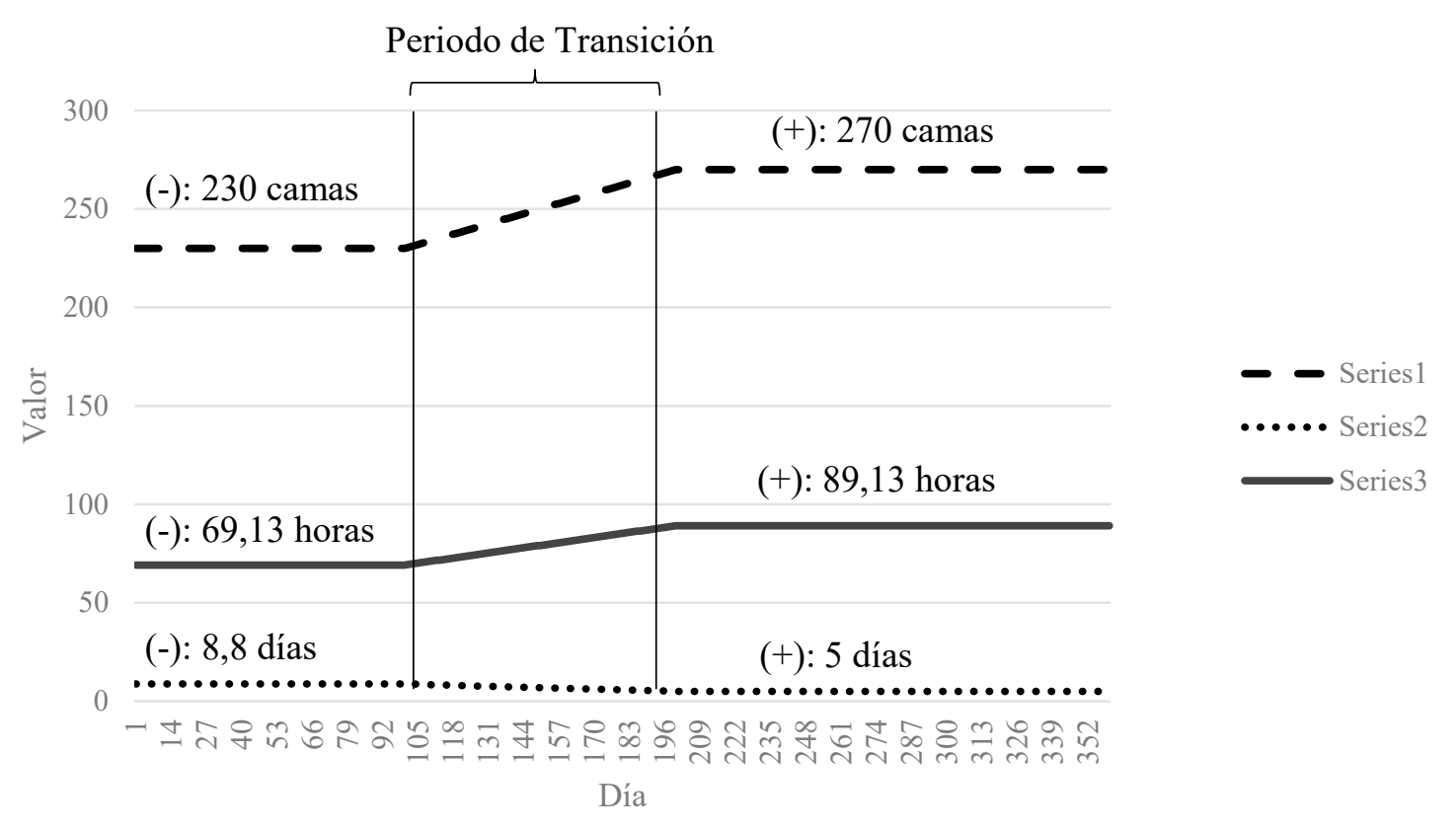

Fig. 7. Cambios implementados para los factores y sus niveles a lo largo del tiempo. Fuente: Autores.

Se generaron diez réplicas de corridas de simulación para cada combinación de factor y nivel completando un total de 80 corridas y en cada una de ellas se contempló un periodo de simulación de 360 días asegurando que el sistema alcanzara un estado estable [38]. Se recolectaron registros para la última observación de las medidas de desempeño bajo análisis: Giro Cama, Porcentaje ocupacional y Tiempo de estancia. Los resultados del experimento fueron analizados como un diseño factorial en donde se aplicaron pruebas ANOVA para establecer si los niveles "Alto" de cada combinación de factores incidió en los resultados de cada medida de desempeño (Tabla 5)

Tabla 5. Resultados de ANOVA para combinaciones de FaCtores y su InCidencia en Medidas DE DESEMPEÑO.

\begin{tabular}{|l|l|l|l|}
\hline & \multicolumn{3}{|c|}{ p-value } \\
\hline Combinación de factores & GCMP & EPM & POM \\
\hline DEP & 0.6432 & 1 & $<2 \mathrm{e}-16$ \\
\hline HMD & 1 & 1 & 1 \\
\hline CDIS & $<2 \mathrm{e}-16$ & 1 & $<2 \mathrm{e}-16$ \\
\hline DEP : HMD & 1 & 1 & 1 \\
\hline DEP : CDIS & 0.9704 & 1 & $<2 \mathrm{e}-16$ \\
\hline HMD : CDIS & 1 & 1 & 1 \\
\hline DEP : HMD : CDIS & 1 & 1 & 1 \\
\hline $\begin{array}{l}\text { Ho: La combinación de factores no incide en el desempeño } \\
\text { Ha: La combinación de factores sí incide en el desempeño }\end{array}$ \\
\hline
\end{tabular}

Fuente: Autores. 
Estos resultados permiten afirmar que mientras que la Estancia Promedio Mensual (EPM) no reacciona a ninguno de los factores establecidos, el Porcentaje Ocupacional (POM) y el Giro Cama Promedio (GCPM) sí lo hacen. En el caso del Porcentaje Ocupacional Mensual (POM), éste tiene cambios significativos al alterar los factores de Duración de la Estancia (DEP), Camas Disponibles (CDIS) y la combinación de estas dos variables (DEP:CDIS).

Para el caso del giro cama, la única alteración significativa se da cuando se modifican las Camas Disponibles (CDIS). La Fig. 8 presenta la forma como el giro cama y el porcentaje ocupacional están reaccionando a los cambios en los factores.
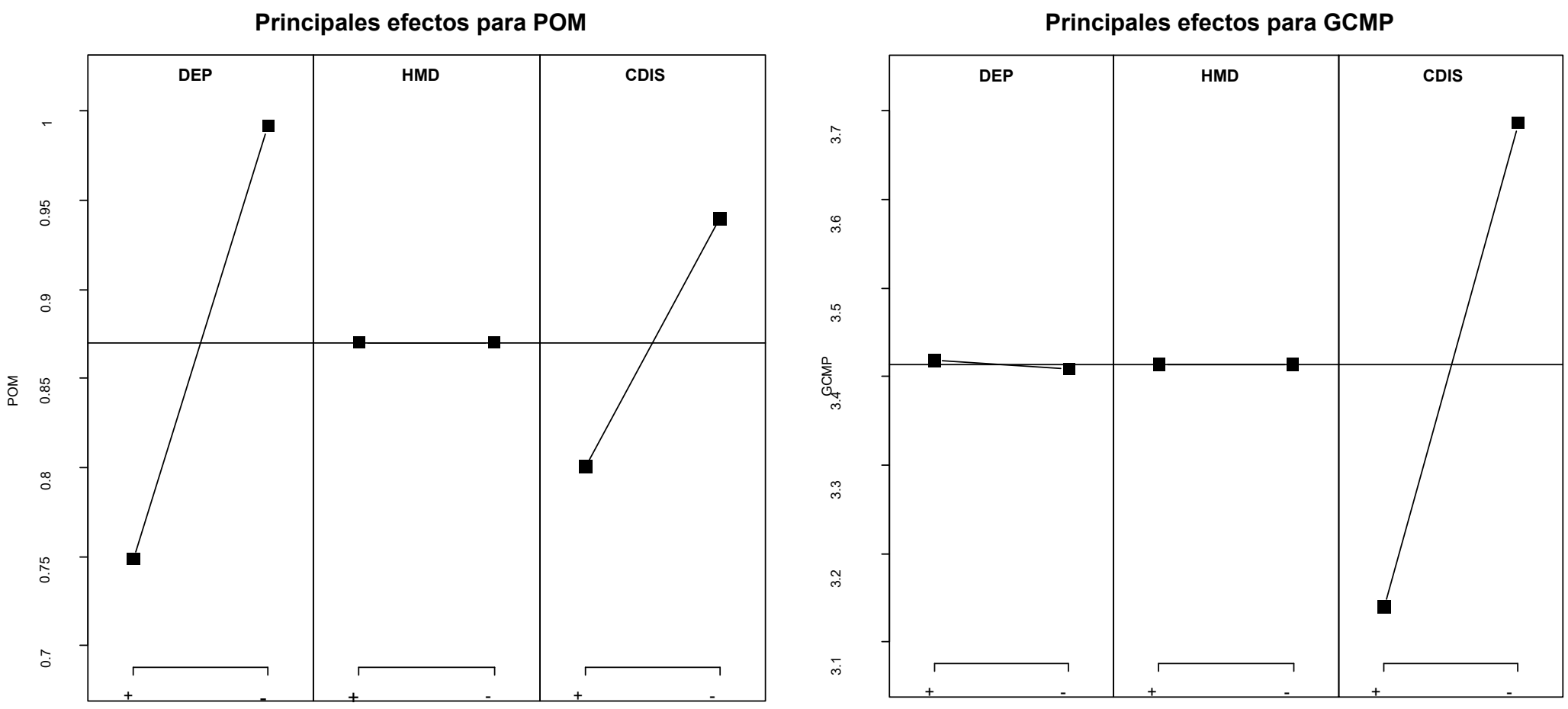

Fig. 8. Comportamiento de los indicadores POM y GCMP ante cambios en los factores. Fuente: Autores.

En la Fig. 8 se denota que el Porcentaje ocupacional es el indicador más sensible a cambios en los factores estudiados. En este sentido se decidió indagar con mayor profundidad acerca de su comportamiento utilizando la técnica de análisis de componentes principales. La Tabla 6 presenta el nivel de variabilidad que los factores considerados (Dim. 1, Dim. 2) lograron recoger para cada uno de los análisis realizados.

TABla 6. Varianza aCUmulada POR ANÁlisis DE COMPONEnTES PRINCIPALES POM.

\begin{tabular}{|l|l|l|l|}
\hline \multicolumn{1}{|c|}{ Experimento } & \% Var DIM. 1 & \% Var DIM. 2 & \% Varianza Acumulada \\
\hline DEP & 65,238 & 33,337 & 98,575 \\
\hline HMD & 42,058 & 32,884 & 74,942 \\
\hline DEP:HMD & 65,238 & 33,337 & 98,575 \\
\hline CDIS & 42,056 & 32,884 & 74,940 \\
\hline DEP:CDIS & 65,238 & 33,337 & 98,575 \\
\hline HMD:CDIS & 42,056 & 32,884 & 74,940 \\
\hline DEP:HMD:CDIS & 65,238 & 33,337 & 98,575 \\
\hline
\end{tabular}

Fuente: Autores.

Se puede evidenciar que los mayores niveles de varianza acumulada se presentan en aquellos experimentos que incluyen la modificación en los días de estancia de pacientes. Esto implica considerar que la estrategia de disminuir los días de estancia promedio tiene un mayor impacto en el indicador de Porcentaje ocupacional mensual. Otros factores como la mejora en las horas médico disponibles o el número de camas instaladas, no reflejaron mayor impacto en la ocupación del sistema.

\section{Conclusiones y RECOMENDACIONES}

La complejidad de un sistema hospitalario pudo ser estudiada utilizando herramientas de simulación, específicamente la Dinámica de sistemas. Bajo esta perspectiva, se pudo examinar la forma como las políticas de gestión de capacidad: manejo de camas, días de estancia 
de pacientes y capacidad del personal; incidieron en las medidas de desempeño de eficiencia hospitalaria global que comúnmente se utilizan en la literatura: giro cama, porcentaje ocupacional y estancia promedio.

La perspectiva de modelar estos sistemas como flujos de pacientes arroja ventajas importantes en la medida en que en un mismo instrumento se pudieron estudiar aspectos asociados a la calidad del servicio, la eficiencia en el uso de recursos y la eficacia en la atención. Esta integración resulta provechosa para la gerencia de estas instituciones, pues brinda una mirada más real y al mismo tiempo holística de la institución hospitalaria. El uso de esta perspectiva integradora resulta novedoso frente a otras formas encontradas en la literatura acerca del modelamiento de sistemas hospitalarios para el estudio de su dimensión logística.

El uso de la simulación continua permitió también comprender la forma como evolucionan los tres indicadores de eficiencia hospitalaria global frente a cambios en variables exógenas o en políticas. El giro cama fue el indicador que respondió con mayor velocidad a los cambios en variables de entrada, mientras que el porcentaje ocupacional lo hizo en segunda instancia. Esta velocidad de respuesta implica que en sistemas reales se deben establecer mecanismos que permitan reconocer de primera mano estos cambios para poder evaluar a tiempo el resultado de estrategias de mejora. Tiene mayor sentido estudiar de cerca el comportamiento del giro cama, que el de tiempo de estancia promedio, para efectos de seguimiento al impacto en políticas de mejora.

La aplicación del análisis de componentes principales en la etapa de experimentación del modelo permitió identificar que el Porcentaje Ocupacional Mensual (POM) es la medida de desempeño que presenta un mayor nivel de dependencia del número de camas y de la duración de la estancia. En segundo plano se encuentra el Giro Cama (GCMP) cuyo comportamiento también resultó sensible a cambios en el número de camas y la duración de la estancia. Como se indicó anteriormente, el Giro cama tuvo una respuesta más rápida que los otros dos indicadores.

Una estrategia adecuada para la gestión de la capacidad hospitalaria consistiría en realizar combinaciones de incremento en la capacidad instalada a partir del número de camas en funcionamiento, acompañado de la implementación de mejoras en la estancia, utilizando metodologías como Lean healthcare para reducir los tiempos de admisión y descarga de pacientes. El monitoreo de estas estrategias debe iniciar con un estricto seguimiento del giro cama en periodos cortos, mientras que el efecto en el porcentaje ocupacional se verá evidenciado con mayor demora. Una mejora en la capacidad de urgencias no tendrá mayor efecto de mejora global en el sistema, si no viene acompañada de las respectivas intervenciones en el número de camas y tiempos de estancia.

Este estudio fue desarrollado de manera longitudinal en una institución hospitalaria. Por lo tanto, se recomienda, para futuros trabajos, desarrollar su aplicación en otro tipo de instituciones siempre y cuando cuenten con la información requerida correctamente sistematizada.

Precisamente la disponibilidad de información sobre indicadores y capacidades fue la mayor dificultad evidenciada en el desarrollo del trabajo. Esta realidad es común a muchas otras entidades y ello dificultaría la implementación de soluciones como la expuesta en este trabajo.

El estudio realizado no consideró algunos aspectos que bien podrían ser tenidos en cuenta para futuros trabajos. En primera instancia, la inclusión de cohortes etarias o discriminación de flujos de pacientes por patología, podría ser simulado en caso de que se contara con información suficiente para este efecto. También resulta importante incorporar otras medidas de capacidad como el número de horas de enfermería disponibles o el tiempo de demora ocasionado por la capacidad de atención en imágenes diagnósticas.

\section{Financiamiento}

Artículo de investigación científica derivado del proyecto de investigación "Mejoramiento de la gestión de operaciones en instituciones hospitalarias en Colombia", desarrollado por el grupo de investigación CINDES y financiado por la Universidad Libre con fecha de inicio: enero de 2014 y en desarrollo a la fecha. 


\section{REFERENCIAS}

[1] M. A. Camacho-Oliveros, Á. E. Morales-Carrillo, M. F. Vega-Novoa \& M. J. Rueda-Varón, "Caracterización de los procesos de atención y flujo de pacientes de la ruta integral de atención en salud maternoperinatal: caso red pública hospitalaria del norte de Cundinamarca", Av. Investig. En Ing., vol. 15, no. 1, pp. 30-47, Dec. 2018. https://doi.org/10.18041/1794-4953/avances.1.1651

[2] S. M. Thompson, R. Day \& R. Garfinkel, "Improving the Flow of Patients Through Healthcare Organizations", in Handbook of Healthcare Operations Management. New York, USA: Springer, 2013, pp. 183-204. https://doi.org/10.1007/978-1-4614-5885-2_7

[3] D. Hernández-Chinchilla, M. Á. Camacho-Oliveros \& E. L. Duarte-Forero, "Análisis del flujo de pacientes en el servicio de urgencias del Hospital Universitario la Samaritana a través de simulación discreta", Av. Investigación en Ing., vol. 14, no. 1, pp. 109-122, Dec. 2017. https://doi.org/10.18041/1794-4953/avances.1.1289

[4] C. Castro, D. Uribe \& J. Castro, "Marco de referencia para el desarrollo de un sistema de apoyo para la toma de decisiones para la gestión de inventarios", IngeCuc, vol. 10, no. 1, pp. 30-42, Jan. 2014.

[5] H. Akkermans \& B. Vos, "Amplification in service supply chains: an exploratory case study from the Telecom industry", Prod. Oper. Manag, vol. 12, no. 2, pp. 204-223, Jan. 2003. https://doi. org/10.1111/j.1937-5956.2003.tb00501.x

[6] T. T. Niranjan \& M. Weaver, "A unifying view of goods and services supply chain management", Serv. Ind. J., vol. 31, no. 14, pp. 2391-2410, Sept. 2010. https://doi.org/10.1080/02642069.2010.504821

[7] D. Dobrzykowski, V. S. Deilami, P. Hong \& S.-C. Kim, "A structured analysis of operations and supply chain management research in healthcare (1982-2011)", Int. J. Prod. Econ., vol. 147, Part B, pp. 514530, Jan. 2014. https://doi.org/10.1016/j.ijpe.2013.04.055

[8] E. Akcali, M. Cote \& C. Lin, "A network flow approach to optimizing hospital bed capacity decisions", Health Care Manag. Sci., vol. 9, no. 4, pp. 391-404, Nov. 2006. https://doi.org/10.1007/s10729-006-0002$\underline{4}$

[9] B. Rechel, S. Wright, J. Barlow \& M. McKee, "Hospital capacity planning: from measuring stocks to modelling flows", Bull. World Health Organ., vol. 88, pp. 632-636, Aug. 2010. https://doi.org/10.2471/ BLT.09.073361

[10] S. Leggat, "Hospital Planning: The Risks of Basing the Future on Past Data", HIMJ, vol. 37, no. 3, pp. 6-14, Feb. 2008. https://doi.org/10.1177/183335830803700302

[11] R. E. Jiménez, "Indicadores de calidad y eficiencia de los servicios hospitalarios: Una mirada actual", Rev. Cuba. Salud Pública, vol. 30, no. 1, Jan. 2004. http://scielo.sld.cu/scielo.php?script=sci arttext\&pid $=\mathrm{S} 0864-34662004000100004$

[12] A. Carreño, "Medición de la calidad, la eficiencia y la productividad en hospitales públicos de tercer nivel de atención en Bogotá,", Univ. Empresa, vol. 11, pp. 203-222, May. 2009.

[13] T. M. Ceballos-Acevedo, P. A. Velásquez-Restrepo \& J. S. Jaén-Posada, "Duración de la estancia hospitalaria. Metodologías para su intervención”, Gerenc. Políticas Salud, vol. 13, no. 27, pp. 274-295, Dec. 2014. Available: https://revistas.javeriana.edu.co/index.php/gerepolsal/article/view/11972

[14] H. Pabon, "Método simplificado para evaluar el desempeño hospitalario", Bol. Oficina Sanit. Panam., vol. 97, no. 1, pp. 33-43, 1984. Disponible en https://iris.paho.org/bitstream/handle/10665.2/16994/ v97n1p33.pdf?sequence=1

[15] L. Socconini, Lean manufacturing paso a paso. Bogotá D.C.: Norma, 2008.

[16] A. Bohorquez, "Desarrollo de una propuesta de mejoramiento continuo para el servicio de urgencias del hospital universitario de La Samaritana (HUS), con la aplicación de la metodología Lean Healthcare", Degree Thesis, Dpto. Ing. Ind, Univ. Libre, Bogotá, D.C., CO, 2017. Available: https://repository.unilibre. edu.co/handle/10901/10592

[17] S. Ruiz \& J. V. Villareal, "Desarrollo de la metodología Lean Healthcare, como estrategia de mejoramiento continuo, que permita elevar el nivel de servicio prestado en el área de Imágenes diagnósticas del hospital Universitario de La Samaritana HUS”, Degree Thesis, Dpto. Ing. Ind, Univ. Libre, Bogotá, D.C., CO, 2018. Available: https://repository.unilibre.edu.co/handle/10901/10570

[18] D. C. Lane \& E. Husemann, "System dynamics mapping of acute patient flows", J. Oper. Res. Soc., vol. 59, no. 2, pp. 213-224, Feb. 2008. https://doi.org/10.1057/palgrave.jors.2602498

[19] L. Green, "Capacity planning and management in hospitals", in Operations research and health care. A handbook of methods and applications. Boston, USA: Kluwer Academic, 2005, pp. 15-42.

[20] J. W. Creswell \& J. D. Creswell, Research design: qualitative, quantitative, and mixed methods approaches, 5th Ed, LA, USA: SAGE, 2018.

[21] M. Schaffernicht, Indagación de situaciones complejas mediante la dinámica de sistemas, vol 1, Talca, CL: Univ. Talca, 2009.

[22] S. Mohiuddin, J. Busby, J. Savović, A. Richards, K. Northstone, W. Hollingworth, J. Donovan \& Ch. Vasilakis, "Patient flow within UK emergency departments: a systematic review of the use of computer simulation modelling methods", BMJ Open, vol. 7, no. 5, May. 2017. https://doi.org/10.1136/bmjopen-2016-015007

[23] J. H. Klein \& T. Young, "Health care: a case of hyper complexity?”, Health Syst., vol. 4, no. 2, pp. 104-110, Dec. 2017. https://doi.org/10.1057/hs.2014.21

[24] D. C. Lane, C. Monefeldt \& J. V. Rosenhead, "Looking in the Wrong Place for Healthcare Improvements: A System Dynamics Study of an Accident and Emergency Department", JORS, vol. 51, no. 5, pp. 518531, Dec. 2000. https://doi.org/10.1057/palgrave.jors.2600892 
[25] T. Rohleder, D. Cooke, P. Rogers, \& J. Egginton, "Coordinating Health Services: An Operations Management Perspective", in Handbook of Healthcare Operations Management. New York, USA: Springer, 2013, pp. 421-445. https://doi.org/10.1007/978-1-4614-5885-2_16

[26] J. B. Homer \& G. B. Hirsch, "System dynamics modeling for public health: background and opportunities", Am. J. Public Health, vol. 96, no. 3, pp. 452-458, Mar. 2006. https://doi.org/10.2105/AJPH.2005.062059 [27] J. Aracil \& F. Gordillo, Dinámica de sistemas. Madrid, ES: Alianza, 1997.

[28] J. D. Sterman, Business Dynamics: Systems thinking and modelling for a complex world. New York, USA: Mc Graw Hill, 2000.

[29] B. Behzad, R. Moraga \& S.-J. Chen, "Modelling healthcare internal service supply chains for the analysis of medication delivery errors and amplification effects", JIEM, vol. 4, no. 4, pp. 554-576, Dec. 2011. https://doi.org/10.3926/jiem.201

[30] M. M. Gunal, "A guide for building hospital simulation models", Health Syst., vol. 1, no. 1, pp. 17-25, Jun. 2012. https://doi.org/10.1057/hs.2012.8

[31] S. Vanderby \& M. W. Carter, "An evaluation of the applicability of system dynamics to patient flow modelling”, J. Oper. Res. Soc., vol. 61, no. 11, pp. 1572-1581, Nov. 2010. https://doi.org/10.1057/jors.2009.150

[32] S. C. Brailsford, V. A. Lattimer, P. Tarnaras y J. C. Turnbull, "Emergency and on-demand health care: modelling a large complex system”, J Oper Res Soc, vol. 55, no. 1, pp. 34-42, Jan. 1990. https://doi. org/10.1057/palgrave.jors.2601667

[33] N. S. Mohd, C.-Y. Liong, A. Y. Md Noh, W. R. Ismail \& N. Ahmad, "Model Sistem Dinamik untuk Meramalkan Bilangan Pesakit dan Keperluan Sumber Tenaga di Zon Kuning Jabatan Kecemasan", USM., vol. 48, no. 4, pp. 909-920, Apr. 2019. https://doi.org/10.17576/jsm-2019-4804-24

[34] S. Jaén, "A Goal-Seeking System Dynamics Methodology for Hospital Bed Capacity Planning", in Applied Computer Sciences in Engineering. New York, USA: Springer, 2018, pp. 280-291, https://doi. org/10.1007/978-3-030-00350-0_24

[35] P. Landa, M. Sonnessa, E. Tànfani \& A. Testi, "System Dynamics Modelling of Emergent and Elective Patient Flows", in Health Care Systems Engineering for Scientists and Practitioners. New York, USA: Springer, 2016, pp. 179-191. https://doi.org/10.1007/978-3-319-35132-2_17

[36] A. V. Esensoy \& M. W. Carter, "High-fidelity whole-system patient flow modeling to assess health care transformation policies", Eur. J. Oper. Res., vol. 266, no. 1, pp. 221-237, Apr. 2018. https://doi.org/10.1016/j. ejor.2017.09.019

[37] H. Rahmandad \& J. D. Sterman, "Reporting guidelines for simulation-based research in social sciences: Reporting Guidelines for Simulation-Based Research", Syst. Dyn. Rev., vol. 28, no. 4, pp. 396-411, Oct. 2012. https://doi.org/10.1002/sdr.1481

[38] Y. Barlas, "Model validation in system dynamics", in Proc. of the Intl. Syst. Dyn. Conf. Scotland, UK: U. Stirling, 1994, pp. 1-10.

Edgar Duarte Forero recibió su título de Ingeniero Industrial de la Universidad del Norte (Barranquilla, Colombia). Posteriormente culminó sus estudios de Maestría en Ingeniería Industrial en la Universidad Distrital Francisco José de Caldas (Bogotá, D.C., Colombia). Actualmente es estudiante del Doctorado en Ingeniería - Industria y organizaciones en la Universidad Nacional (Colombia). Se desempeña como docente de la Facultad de Ingeniería de la Universidad Libre y es miembro del grupo de investigación CINDES. https://orcid.org/00000001-8072-6976

Manuel Ángel Camacho Oliveros es Ingeniero Industrial de la Universidad Distrital Francisco José de Caldas (Bogotá, D.C., Colombia). Especialista en Ingeniería de Producción de la Universidad Distrital Francisco José de Caldas (Bogotá, D.C., Colombia). Realizó una Maestría en Sistema de Calidad y Productividad en el Instituto Tecnológico y de Estudios Superiores de Monterrey de México (Monterrey, México). Actualmente es Doctorando en Ingeniería de Procesos en la Universidad EAN (Bogotá, D.C., Colombia). Se desempeña como docente investigador de la facultad de Ingeniería de la Universidad Libre (Bogotá, D.C., Colombia) y es integrantes del grupo de investigación CINDES de la misma institución. https://orcid.org/0000-0001-7449$\underline{2181}$ 